\title{
Gap between risk factors and prevention strategies? A national survey of falls prevention among medical and surgical patients in China
}

\author{
Lixia Xia ${ }^{1}$, Yi Zheng ${ }^{1}$, Zheng Lin $^{1}$, Peng Chen ${ }^{2}$, Ke Mei ${ }^{1}$, Jing Zhao ${ }^{1}$, Yi Liu ${ }^{1}$, Bao Song ${ }^{1}$, \\ Hong Gao ${ }^{1}$, Chao Sun ${ }^{1}$, Hui Yang ${ }^{1}$, Ying Wang ${ }^{1}$, Kui Song ${ }^{1}$, Yan Yang ${ }^{1}$, Xiao Luan ${ }^{1}$, \\ Xianxiu Wen ${ }^{1}$, Xin Yin ${ }^{1}, \mathrm{~A} \mathrm{Fu}^{1}$, Ying $\mathrm{Cai}^{1}, \mathrm{Li} \mathrm{Xie}^{1}$, Ya Li ${ }^{1}$, Xi Dai ${ }^{1}$, Jieyu Lu ${ }^{1}, \mathrm{Ze} \mathrm{Gu}^{1}$, \\ Rong $\mathrm{Wang}^{1}$, and $\mathrm{Xin} \mathrm{Wu}^{1}$ \\ ${ }^{1}$ Affiliation not available \\ ${ }^{2}$ The First Affiliated Hospital with Nanjing Medical University
}

September 9, 2020

\begin{abstract}
Abstract Rationale, Aims, and Objectives: To determine the extent to which nurses reportedly assess evidence-based falls risk factors and identify targeted prevention implemented for medical and surgical patients in China. Method: This was a multicenter retrospective observational study. Respondents were nurses working in medical and surgical units of Chinese 662 hospitals. Fall-risk factors assessments and targeted interventions by health professionals were collected online by the Nursing Management Committee of Chinese Nursing Association in China, in 2019. Results: A total of 68527 valid questionnaires were recovered (95.0\%). In medical and surgical units, nurses were most likely to report assessing balance, mobility and strength $(81.6 \%)$ and orthostatic hypotension $(76.4 \%)$ for falls and least likely to continence $(61.3 \%)$ and feet and footwear (55.8\%). Appropriate footwear (79.3\%) and managing syncope, dizziness and vertigo (73.8\%) were the most frequently falls multiple interventions, while managing postural hypotension (48.8\%) and cognitive imparement (48.4\%) were the least. Nine fall-risk factors with clearly matched multifactorial interventions were determined in medical and surgical units (68.2\% 97.5\%). On comparative analysis, Cognitive therapy, Toileting-assistance, Indwelling catheter, Monitor postural blood pressure, Dizziness and vertigo interventions, Minimise asthma triggers, Educating medication use, Keeping floor surface clean and dry were more common in medical ward, while With the head of the bed raised, Assessments and referrals and Bed brakes locked in surgical ward. Conclusion: Health professionals are generally concerned about risk factors and prevention for falls with their patients in much of China, however limited attention was directed at the continence, feet and footwears assessment and managing cognitive imparement. Evidence-based prevention should be further tailored to the individual, based on an individual risk factors.
\end{abstract}

\section{Hosted file}

Gap between risk factors and prevention strategies? A national survey of falls prevention among medical available at https://authorea.com/users/357613/articles/480108-gap-between-risk-factors-andprevention-strategies-a-national-survey-of-falls-prevention-among-medical-and-surgicalpatients-in-china 


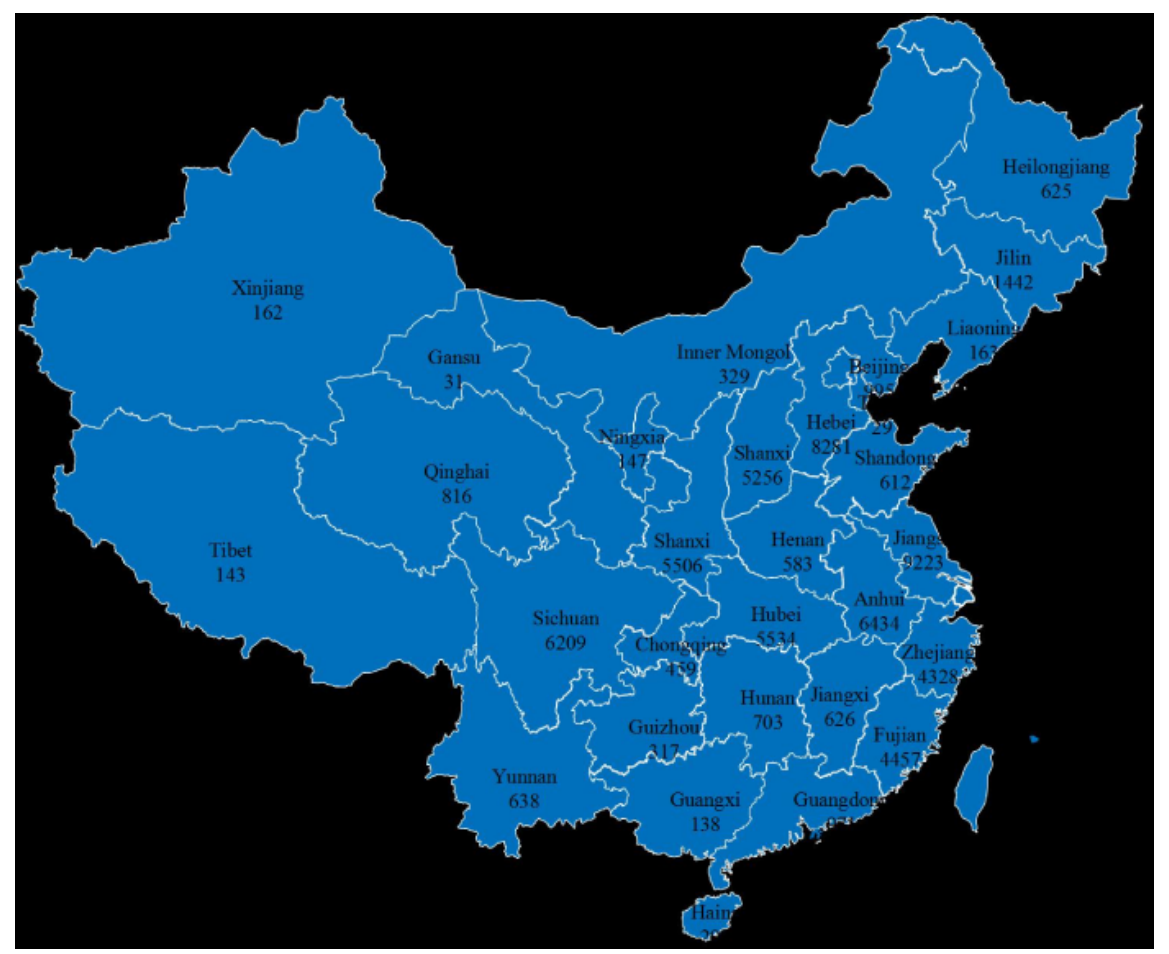

Appropriate footwear

Manage syncope, dizziness and vertigo

Medication management

Manage balance, mobility and strength

Environmental modification

Other interventions (e.g. restraints)

Continence management

Vision interventions

Manage postural hypotension

Manage cognitive imparement
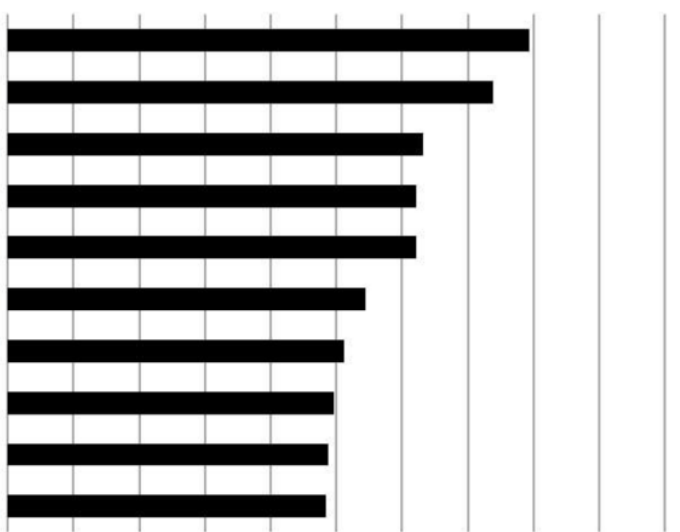

$\begin{array}{llllllll}0 & 10 & 20 & 30 & 40 & 50 & 60 & 70\end{array}$

$\begin{array}{lll}80 & 90 & 100\end{array}$

Percentage (\%) 